\title{
Optimal inventory management in a fluctuating market
}

\author{
De Cuypere E., De Turck K., Bruneel H., and Fiems D. \\ Department of Telecommunications and Information Processing, Ghent University, \\ St-Pietersnieuwstraat 41, B-9000, Belgium \\ (eline.decuypere, koen.deturck, herwig.bruneel, \\ dieter.fiems)@telin.ugent.be
}

\begin{abstract}
In the highly competitive environment in which companies operate today, it is crucial that the supporting processes such as inventory management are as efficient as possible. In particular, a trade-off between inventory costs and service levels needs to be assessed. In this paper, we determine an optimal batch ordering policy accounting for both demand and market price fluctuations such that the long-term discounted cost is minimised. This means that future costs are multiplied by a constant factor smaller than one as we need to take inflation and other factors into account. To this end, the inventory system is modelled as a Markovian queueing system with finite capacity in a random environment. Assuming geometrically distributed lead times, Markovian demand and price fluctuations, the optimal ordering strategy is determined by a Markov decision process (MDP) approach. To illustrate our results, we analyse the ordering policy under several price fluctuation scenarios by some numerical examples.
\end{abstract}

\section{Introduction}

The impact of demand fluctuations has been extensively studied as an extension of the classical EOQ model [8]. Resh et al. and Donaldson established an algorithm to determine the optimal order quantity and the timing for a linearly increasing demand pattern $[12,4]$. Barbosa and Friedman then generalised the solutions for various, similar demand models [1]. Henery extended the demand to any log-concave demand function [6]. Yan and Kulkarni developed an inventory system in which the stochastic demand is assumed to be a continuous-time Markov chain [18]. The lead time equals zero and an ordering is only possible when the inventory is empty. Song and Zipkin determined optimal inventory policies in a fluctuating demand environment [13]. In particular, the demand process is described as a Markov-modulated Poisson process and the lead times are assumed to be phase-type distributed. Chien et. al aimed at solving capacity expansion and migration decision problems for a semiconductor industry with stochastic capacity expansion lead times. Also, the demand processes of different products are modelled by a discrete time non-homogeneous Markov chain [3]. 
In contrast to fluctuations in demand, the impact of price fluctuations is a rather unexplored scientific domain. J.-T. Teng et. al have established an algorithm assuming both fluctuating demand and price. However, this algorithm relates to products with a continuously decreasing price, such as high-technology products, and no lead time is assumed for delivery [16].

In this paper, we propose a discrete-time Markovian model with stochastic lead times that accounts for both demand and market price fluctuations. Note that modelling the system as a DTCM instead of a CTMC simplifies significantly the analysis. Indeed, we assume that events, such as an order delivery or a product demand, may only happen at discrete time points. The lead time, i.e. the time between two consecutive deliveries, follows a geometrical distribution. With regard to demand fluctuations, the model is relevant for products in their maturity phase where customer demand levels off. Here, we assume that demand follows a Bernoulli distribution, meaning that the time between two consecutive demands is geometrically distributed. With regard to price fluctuations, we focus on medium-term price fluctuations around a long-term average. Based on the defined Markovian model, we aim at determining an optimal ordering policy by means of the theory of the Markov decision process (MDP).

Our paper is organised as follows. Section 2 describes the inventory model at hand. In particular, the inventory system is modelled as a three-dimensional Markov chain and the optimal ordering policy is determined by the theory of the Markov decision process. Also, the solution methods are explained. To illustrate our approach, section 3 considers some numerical examples. In particular, we determine the optimal ordering policy under several price fluctuation scenarios. Finally, conclusions are drawn in section 4.

\section{Model description}

We consider the inventory system of a product subject to demand as well as price fluctuations. Furthermore, the lead time of this product is stochastic and demand is lost when the inventory is empty, i.e. there is no backlogging. Finally, there is at most one ongoing order and a customer demand always consists of one item. In this section, the assumptions and the research approach are explained.

\subsection{Assumptions}

Concerning the lead time and the market fluctuations, we assume that:

- the time between two consecutive order deliveries is geometrically distributed with parameter $\kappa$,

- the demand is Bernoulli distributed with parameter $p$. This means that at each discrete time unit the inventory level decreases by one or remains the same with probability $p$ and $1-p$ respectively,

- the price fluctuates around a long-term average with frequency $\alpha$ and $\beta$ and with variability $v$. The parameters $\alpha$ and $\beta$ denote the probability that 
the price decreases and increases respectively and $v$ is the rate at which $\alpha$ and $\beta$ decrease as the price moves further away from the average price. The probability that the price level remains unchanged at the next time unit is thus equal to $1-(\alpha+\beta)$ and is therefore independent of the current price level.

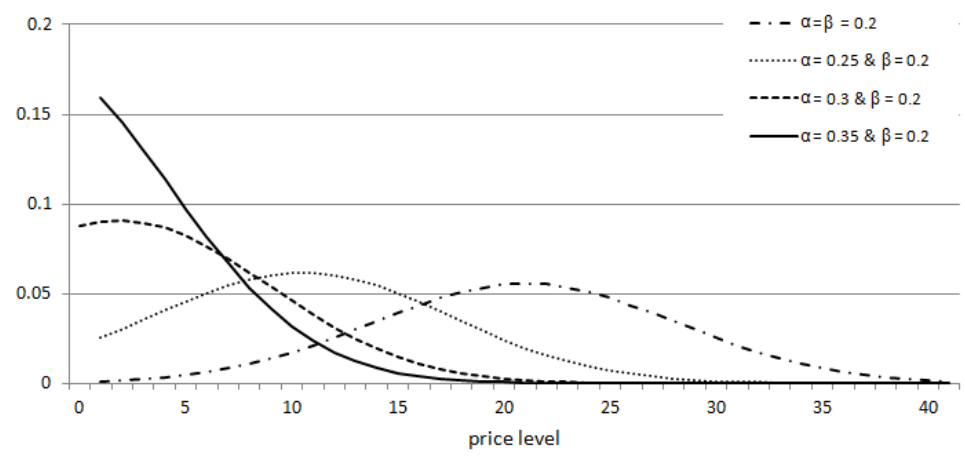

Fig. 1. Price distributions in function of $\alpha$ and $\beta$.

Figure 1 depicts the price distribution in function of $\alpha$ and $\beta$, in the case that there are $K=40$ possible price levels. As expected, the probability distribution shifts to the left as the probability to have a price decrease increases. This also means that the average price level decreases. Another effect of an increase in $\alpha$ is that the steady-state probability to have a relatively low price (e.g. 5) increases.

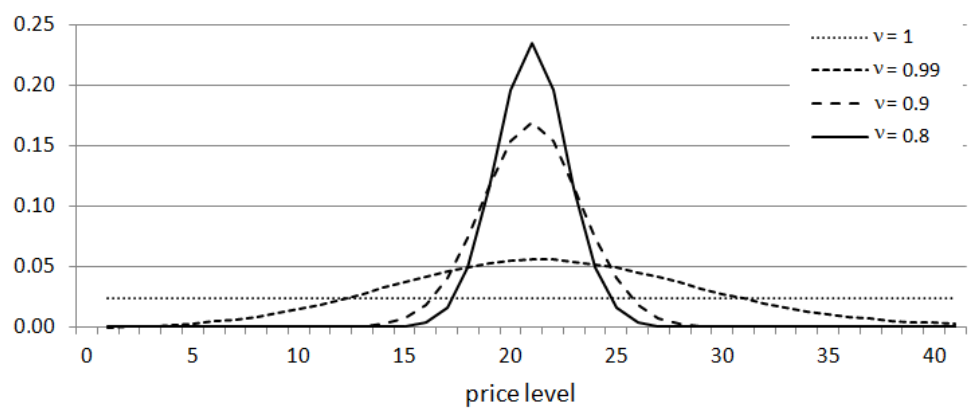

Fig. 2. Price distributions in function of $v$.

Figure 2 depicts the steady-state price probability versus the price level for different values of $v$. As the figure shows, the smaller the rate $v$, the higher the 
probability that the price level is in the vicinity of the long-term average and the smaller the probability that an extreme (high or low) price level is reached. However, if the rate is high, the probability of an extreme price level is relatively high. Finally, if the probability of a price decrease and increase are independent of the current price level, i.e. $v=1$, the probability distribution of the price is represented by a straight line.

Finally, to determine an optimal ordering policy we need to minimise the total cost. Based on the (EOQ) economic order quantity model developed by F.W. Harris, we consider the following total cost function [5]:

$$
\mathrm{C}_{t}=C_{h} \frac{Q}{2}+C_{0}+Q P_{t}+C_{v} \mathbb{1}_{\{n=-1\}}
$$

where $C_{o}$ and $C_{h}$ are the cost per order and the inventory cost per unit respectively, $Q$ is equal to the order quantity and $P_{t}$ is the price at the time of ordering $t$. The first cost is assumed to be proportional to the mean average of goods stored in the inventory $\frac{Q}{2}$ and includes the opportunity cost of fixed capital and the cost for storage and internal transport of the goods. This cost is often the largest proportion of the total cost and have varied between 6 and $18 \%$ during the last 25 years [10]. We assume that the inventory cost is proportional to the inventory level. The second is only relevant when an order is placed and includes a fixed order cost $C_{o}$ and a variable purchase cost per order $Q P_{t}$. In contrast with the inventory costs, the purchase cost depends on the price and the order quantity but not on the current inventory level. The last $\operatorname{cost} C_{v}$ is the cost due to lost demand. This is the case when the demand is not satisfied because the inventory is empty. This cost assumption leads to the introduction of a fictional inventory level $n=-1$ in the model. If the inventory is empty, a demand at time $t$ leads to a system in inventory -1 at time $t+1$ and the cost is added to the total cost. An inventory system in this fictional level behaves in the same way as a system with an empty inventory. At time $t+2$, the system returns to an inventory level equal to zero if there is no demand and remains at level -1 when there is a new demand. Note that this cost can be seen as the sum of several factors, such as the cost due to lost sales and the damage on the industry's reputation.

\subsection{Research approach}

Three-dimensional discrete-time Markov chain The assumptions made above allow us to model the inventory system as a stationary Markov chain. In particular, we describe the inventory system as a three-dimensional discretetime Markov chain with finite state space $\mathcal{S}=\mathcal{C} \times \mathcal{K} \times \mathcal{O}$ with $\mathcal{C}=\{-1, \ldots, C\}$ where $C$ is the buffer capacity of the inventory system, $\mathcal{K}=\{1,2 \ldots, K\}$ where $K$ is the number of possible price levels and $\mathcal{O}=\{0, \ldots, C\}$. The state of the inventory system $S_{t}$ at a discrete time $t$ is described by the triplet $[n, m, i], n$ being the inventory level, $m$ being the price level and $i$ being the ordering in process that indicates whether or not an order is placed but not yet delivered, 
and also how many units are ordered. In particular, if no order is in process, then the system is in state 0 and if an order is in process, then the state is equal to $x=\{1, \ldots, C\}$, being equal to the order quantity that has been ordered.

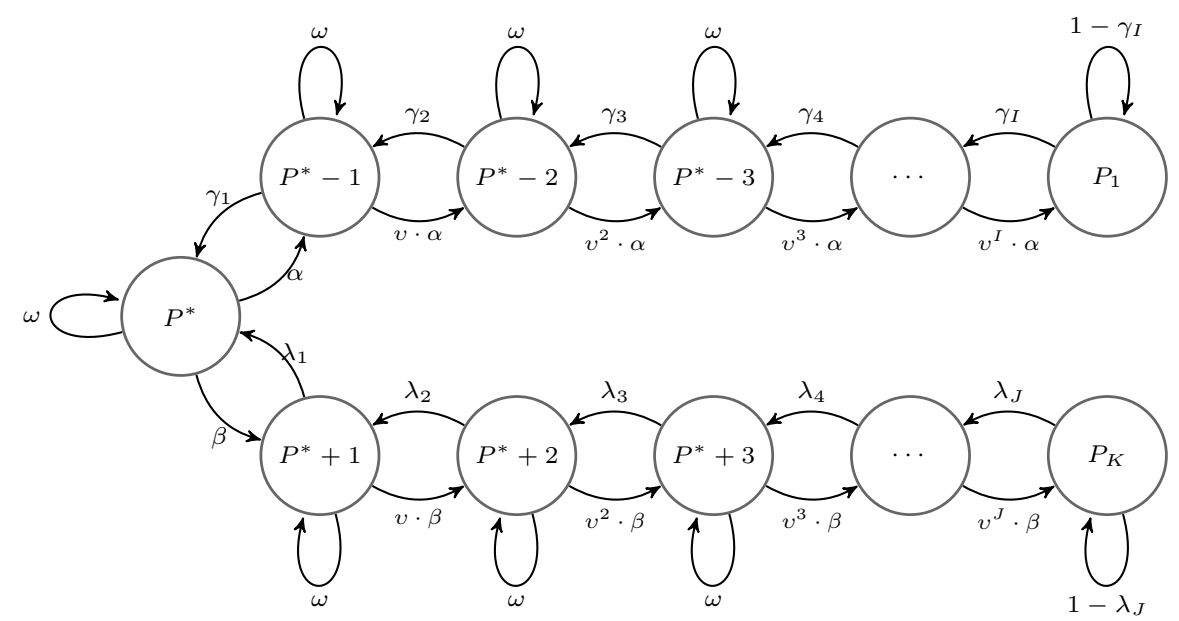

Fig. 3. State diagram of the price level

Figure 3 depicts the state diagram of the price level with an average price $P^{*}$. As previously mentioned, we assume a range of $K$ possible price levels $P=$ $\left(P_{1}, P_{2}, \ldots, P_{K}\right)$ and a decrease of the probability of a price increase $\beta$ and a price decrease $\alpha$ with rate $v$ as the price level moves further from the price average $P^{*}$. This means that the probability that the price further deviates from the average price decreases at each step with rate $v$. If this was not the case, the price probabilities would be independent of the state in which the system is and the steady-state probabilities of all the price levels would be equal, this being an unrealistic assumption according to us. The parameters in figure 3 are defined as follows:

$$
\begin{aligned}
& -\omega=1-(\alpha+\beta) \\
& -\gamma_{i}=\beta+\left(\alpha-v^{i} \alpha\right) \\
& -\lambda_{j}=\alpha+\left(\beta-v^{j} \beta\right)
\end{aligned}
$$

where $i=1, \ldots, I$ and $j=1, \ldots, J$. The parameter $\omega$ is the probability that the price remains at the same price level and the parameter $\gamma_{i}$ and $\lambda_{j}$ represent the probability of a price increase and decrease after $i$ price decreases and $j$ price increases, respectively.

Markov decision process A Markov decision process (MDP) is very similar to a Markov chain in the sense that the evolution of a system is described by a series 
of transitions in a set of predefined states. However, the decision process differs from a Markov chain as the transition matrix now depends on the action taken by the decision maker. A cost is attached at each combination of state and action. The goal is to find an optimal policy which take both immediate and future costs into account. A commonly used cost criterion is the minimisation of the long-term expected average cost per unit time. Another way is to minimise the expected total discounted cost with a discount factor $d$. Both criteria are suitable for our purposes since we work with an infinite horizon. However, we choose to find our optimal policy based on the total discounted cost. The motivation and the explanation of this cost model is given below.

By means of the theory of a Markov decision process, we define the tuple $\left(\mathcal{S}, \mathcal{A}, P_{a}, R_{a}\right)$ being:

- the finite state space $\mathcal{S}=\mathcal{C} \times \mathcal{K} \times \mathcal{O}$ of our discrete-time Markov chain with $\mathcal{C}=\{-1, \ldots, C\}, \mathcal{K}=\{1,2 \ldots, K\}$ and $\mathcal{O}=\{0, \ldots, C\}$,

- the set of actions $d_{s} \in \mathcal{A}=\{0,1, \ldots, C\}$,

- the transition matrix $P_{a}\left(s, s^{\prime}\right)=\operatorname{Pr}\left[S_{t+1}=s^{\prime} \mid S_{t}=s, a_{t}=a\right]$,

- the cost matrix $R_{a}\left(s, s^{\prime}\right)=R_{a}(s)$.

where $S_{t}$ and $S_{t+1}$ represent respectively the state of the system at time $t$ and $t+1$. In a Markov decision process, action $a=0$ means that no order is placed and action $a=x=\{1, \ldots, C\}$ means that an order of $x$ units is placed. The chosen action influences the transition probabilities, the immediate and the future costs as well as the behaviour of the system in the future.

Then, there are two types of matrices of interest. The first one, the transition matrices $P_{a}\left(s, s^{\prime}\right)$, give the probability to reach $s^{\prime}$ at time $t+1$ when the system is in state $s$ and action $a$ has been undertaken by the decision maker. The second one, the cost matrices $R_{a}\left(s, s^{\prime}\right)$ give the cost obtained when the system is in state $s^{\prime}$ at time $t+1$ after choosing action $a$ in state $s$. The cost matrix can be written as $R_{a}(s)$ because the cost is independent of the state in which the system ends up at time $t+1$. In particular, the inventory cost depends on the current inventory level and the purchase cost depends on the chosen action (and consequently on the order quantity) and on the current price.

Finally, after having defined our Markov decision process, we aim at finding an optimal action at each state such that the total discounted cost is minimised. The discounted cost is defined as,

$$
\sum_{t=0}^{\infty} d^{t} R_{a}\left(S_{t}\right), d=\frac{1}{1+r}, 0<d \leq 1
$$

where $r$ is the current interest rate and $d^{t}$ is equal to the current value of one unit-cost after $t$ time periods. The value of this factor, typically close to 1 , plays an important role in the model [7]. If the costs are greatly discounted, i.e. the discount factor has a low value, the immediate ordering costs have much more weight than the future inventory costs. Hence, the order quantity will be larger such that the ordering cost can be assigned to a larger number of units. 
Furthermore, the value of the discount rate depends on the specific situation of the company and of the size of the time interval. Indeed, the cost will be much more discounted for a time interval of one month than of a day.

According to [14], it is appropriate to apply the discounted cost if the time intervals of the modelled Markov chain are sufficiently long. As we consider goods with a low turnover and medium-term price fluctuations, the discounted cost model can be applied.

Although it is theoretically possible to solve a Markov decision process by calculating the total cost for each possible policy, it becomes infeasible for larger problems. Several algorithms are available to find the optimal ordering policy in a more efficient way. In this work, five different solution methods are used and compared in order to address the most efficient method for our specific model. These methods are linear programming, policy iteration, value iteration and a modified algorithm of both policy iteration and value iteration.

\section{Solution methods}

Linear programming It is possible to solve a Markov decision process with the aid of linear programming. This method uses a mathematical model for describing a problem and determining the best outcome by optimising a linear objective function subject to linear constraints. We use the simplex method to find our optimal deterministic policy. Further details of this method can be found in [7].

Dynamic programming Besides linear programming, Markov decision processes can also be solved with the aid of dynamic programming. To find the optimal policy of a Markov decision process, there are two main functions that are solved iteratively for each state $s$. First, the policy $\phi(s)$ for each state $s$ represents the order quantity when the system is in state $s$. Second, the variable $V^{\phi}(s)$ is the 'value' of a given state $s$ if a well-defined policy $\phi(s)$ is followed. This variable is calculated for each state $s$, which leads to a system of equations. The step in which this system is solved is called 'value determination'. The optimum policy is denoted as $\phi^{*}$ and is thus the policy for which the values $V^{\phi^{*}}(s)$ are smaller than or equal to any other values of the ordering policies in every possible state $s$. Note that we aim at finding the smallest value for each state $s$ as the value is calculated based on costs and not on rewards. In the formulas below, the parameter $k$ represents the successive iterations [14,17]. In other words, $k+1$ is an indication for the next iteration.

$$
\begin{aligned}
& \phi(s)=\underset{\mathrm{a}}{\operatorname{argmin}}\left\{\sum_{s^{\prime}} P_{a}\left(s, s^{\prime}\right)\left(R_{a}\left(s, s^{\prime}\right)+d V\left(s^{\prime}\right)\right\}\right. \\
& V_{k+1}(s)=\sum_{s^{\prime}} P_{\phi(s)}\left(s, s^{\prime}\right)\left(R_{\phi(s)}\left(s, s^{\prime}\right)+d V_{k}\left(s^{\prime}\right)\right)
\end{aligned}
$$


The value function $V(s)$ is the expected total discounted cost when the system starts in state $s$ where policy $\phi$ is applied and evolves to infinity. The formula (3) includes as many equations as there are states being the unknowns.

Several algorithms make use of these two steps. The order in which these steps are carried out depends on the particular variant of the algorithm. The different solution methods are now explained further in details.

Policy iteration A popular method of dynamic programming is the 'policy improvement algorithm' or 'policy iteration', in which the policy iteratively improves [9]. The main advantage of this method is the high efficiency. In particular, a much smaller number of iterations is required to reach the optimal solution of a Markov decision process compared to the simplex method in linear programming.

The first step in this algorithm is to choose an arbitrary policy $\phi_{0}$. In other words, an arbitrary action $a_{s}$ is selected for each state $s$. Then, in the value determination - also called the value evaluation — step, a series of equations are solved for each state $s$ as in formula (2). In other words, a value $V^{\phi_{0}}$ is calculated for each state $s$. Based on this result an improved policy $\phi_{1}$ is set in the policy improvement step as shown in formula (3). This process corresponds to one iteration. The equations $V_{1}(s)$ of the valuation determination step is set and developed, after which a new improved policy $\phi_{2}$ is given. This iterative process is repeated until two successive iterations yield an identical policy. The policy $\phi^{*}$ is defined as the optimal ordering policy. Figure 4 shows the interaction between the policy and the value function graphically. Here, $E$, represents the value evaluation step and $I$ represents the policy improvement step.

In this solution method, there is a stop criterion. The algorithm is complete if $\phi$ does not change when using the formula (2) in the policy improvement step. Moreover, the algorithm guarantees an optimal solution in a finite number of iterations since the number of possibilities for the ordering policy is finite. Also note that each policy evaluation $V_{k+1}(s)$ starts from the value function $V_{k}\left(s^{\prime}\right)$ of the previous iteration, resulting in a very fast convergence speed. Further details on this algorithm can be found in [15], [17] and [7].

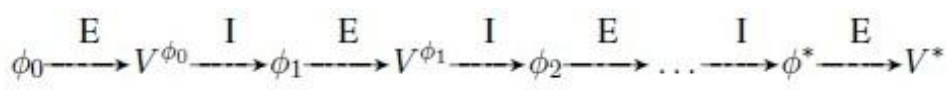

Fig. 4. Policy iteration

Value iteration A disadvantage of policy iteration is that each iteration in the policy evaluation step requires iterative calculations in itself. Moreover, convergence takes place only in the limit, i.e. if the policy is the same in two successive iterations. The question is whether we can stop the iteration process earlier and 
still find the optimal ordering policy. A possibility is to perform the policy evaluation step in each iteration only once for each state $s$ and not until convergence occurs [15]. This popular solution method, developed by Bellman, is called 'value iteration' [2]. This is a method based on the principle of backward induction. In this algorithm, the so-called 'Bellman equation' is used instead of the $\phi$-sequence, which is obtained by substituting the formula for $\phi(s)$ in the formula for $V(s)$.

$$
\begin{aligned}
V_{k}(s)= & \min _{\mathrm{a}}\left\{\mathrm{E}\left[r_{t+1}+d V_{k}\left(s_{t+1}\right) \mid s_{t}=s, a_{t}=a\right]\right\} \\
& =\min _{\mathrm{a}}\left\{\sum_{s^{\prime}} P_{a}\left(s, s^{\prime}\right)\left(R_{a}\left(s, s^{\prime}\right)+d V_{k}\left(s^{\prime}\right)\right)\right\}
\end{aligned}
$$

In other words, the value of a certain state is defined as the sum of the minimum expected cost in that state and the expected discounted value of all possible states $s^{\prime}$ that can be achieved from state $s$. The value iteration algorithm consists of iteratively solving the Bellman equation for each state $s$. In theory, an infinite number of iterations is needed to find the optimal values $V^{*}$. In practice, the algorithm is completed when an epsilon-optimal solution is found. A disadvantage of the value iteration algorithm is that a solution is found only at the end of the iterations, in contrast to policy iteration where the solution is systematically improved, which is more transparent. For very large problems, a decent, but not optimal, solution can be found in a reasonable time with policy iteration while this is impossible with value iteration. A more detailed explanation on this algorithm can also be found in [17] and [7].

Modified policy iteration The 'modified policy iteration' is, as the name suggests, a slightly modified version of the policy iteration algorithm. In the original algorithm, the first step, i.e. the policy improvement, iterates once, while the second step, i.e. the policy evaluation, is repeated until it converges.

In the modified version, the first step is the same and the second step is repeated only a limited number of times and thus not until convergence occurs. In particular, a number of value iteration steps are performed such that a reasonably good approximation of the solution is given whereas an exact solution is given in the original algorithm. In addition, the algorithm is completed when an epsilonoptimal solution is found, in contrast to the original algorithm where there is a strict stop criterion for optimality, i.e. if the policy is the same in two successive iterations [11]. To summarise, the modified policy iteration algorithm is similar to the standard policy iteration algorithm except that the policy evaluation step is calculated by using the value iteration algorithm and the algorithm stops when an epsilon-solution is found.

Gauss-Seidel value iteration The adapted version of the value iteration algorithm that will be used in this work is the 'Gauss-Seidel value iteration' algorithm. As for the original algorithm, the Bellman equation is iteratively solved, but in this variant $V_{k+1}(s)$ is used instead of $V_{k}(s)$. 


\section{$3 \quad$ Numerical results}

\section{Markovian demand and price fluctuations}

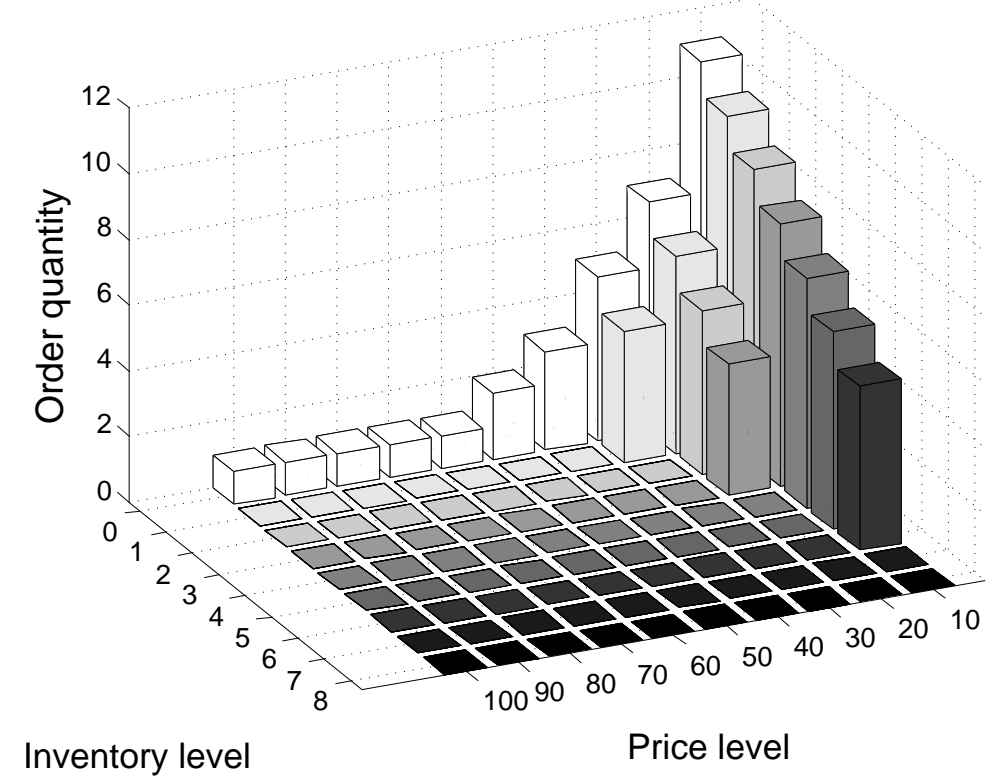

Fig. 5. Optimal ordering policy at a given inventory- and price level and with immediate delivery.

The first numerical example considers an inventory system with immediate delivery, i.e. the probability to have a delivery $\kappa$ equals 1 . In particular, figure 5 depicts the optimal ordering policy for a combined inventory- and price level. The inventory system has a buffer capacity $C$ equal to 15 and we assume a price range of 10 levels (from 10 to 100). The holding cost $C_{h}$ equals 0.1 and the fixed ordering $\operatorname{cost} C_{o}$ equals 20 . Concerning the price fluctuations, the probability of a price decrease and increase, respectively $\alpha$ and $\beta$, equal 0.4 and the variability is defined by the parameter $v$ equal to 0.2 . The probability to have a demand of one unit $p$ equals 0.6 and we assume a discount factor $d$ equal to 0.9 . Finally, to ensure that an order is placed when the inventory is empty, we assume a very high cost due lost demand, i.e. $C_{v}$ equals 1000.

As expected, the lower the inventory- and the price level, the larger the order quantity. The order quantity as well as the threshold at which an order is placed depends on several factors such as the ordering costs, the inventory costs and the price fluctuations. Moreover, each inventory level is characterised by an exponential function with the order quantity as dependent variable and the price 
level as independent variable. In the higher inventory levels, this exponential function is earlier interrupted because it is possible to wait for a decreased price in the near future such that the holding costs and the purchase costs are kept low. The same is observed when the inventory level is the independent variable. For higher price levels, the decreasing function is earlier interrupted due to the combined effect of the inventory- and purchase costs. Finally, the decision maker should not order in the vast majority of the states. This is explained by the low uncertainty inherent in the model due to the absence of an uncertain lead time. If the price level is high, we will always wait because the probability of a price decrease is real and there is no risk that the demand is not satisfied due to an empty inventory. If a lead time is introduced, the risk of an unsatisfied demand is present and the decision maker will have to handle faster and will sometimes even be forced to order at a very high price. A numerical example of this scenario is given in the next paragraph.

\section{Geometrically distributed lead times}

Both figures 6(a) and 6(b) quantify the impact of the cost due to lost demand on the ordering policy. In both figures, the parameters have the same value as in the previous section and we assume that the time between two consecutive order deliveries is geometrically distributed with $\kappa$ equal to 0.5 . In figure 6(a) and $6(\mathrm{~b})$, the cost due to lost demand $C_{v}$ equals 100 and 1000 respectively.

As figure 6(a) shows, no orders are placed at a high price even if the inventory is empty. This is due to the fact that the cost due to lost demand does not outweigh the high ordering cost. However, in figure $6(\mathrm{~b})$, where the cost due to lost demand is multiplied by 10 , an order is placed even at a very high price and not only when the inventory is empty. In this case, an inventory shortage is avoided at all times. As expected, the combination of a low probability to have a delivery with a high cost due to lost demand would enhance further this effect.

\section{Comparison between the different solution methods}

In this section, we aim at comparing the different solution method algorithms explained in the section 'Solution methods'. Figure 7 summarises the performance of the various solution methods as a function of the number of states.

As the figure shows, the linear programming algorithm is not suitable for our model with a large state space. The reason is that this solution method is not specifically developed to solve Markov decision processes, while it is the case for the other four methods. Furthermore, the value iteration and its derivative Gauss-Seidel algorithm rise exponentially with large state spaces. Moreover, the standard value iteration algorithm is much more efficient than the Gauss-Seidel approximation, probably due to a lower number of iterations. Furthermore, we can observe that the state space has to be very large before the value iteration algorithm performs significantly worse than the policy iteration. The modified policy iteration algorithm is in turn slightly more efficient than the standard 

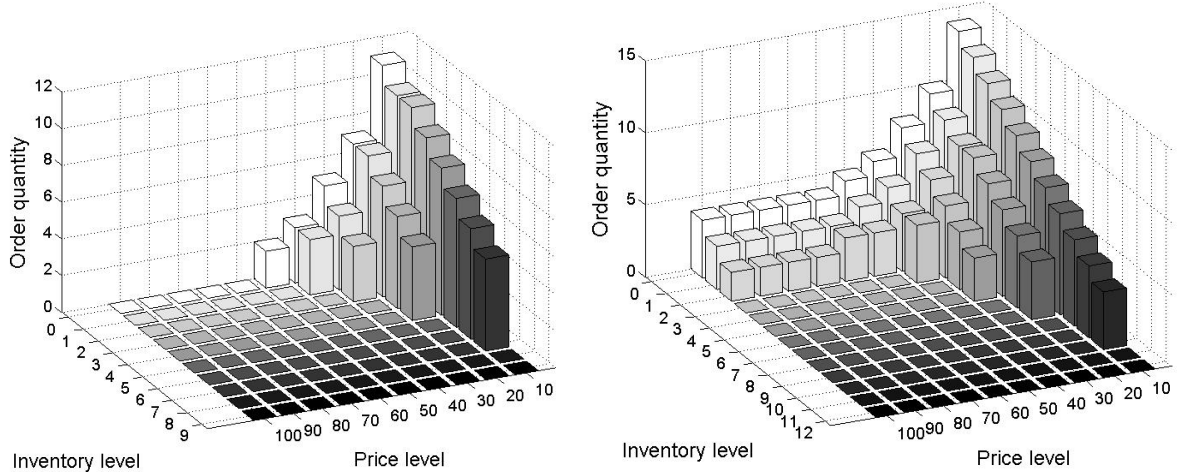

Fig. 6. Impact of the cost due to lost demand on the optimal ordering policy $\left(C_{v}=100\right.$ and $C_{v}=1000$ in the left- and right figure respectively).

policy iteration algorithm, but the difference is negligible. Finally, we may conclude that the modified policy iteration algorithm is the most efficient solution method for this specific model.

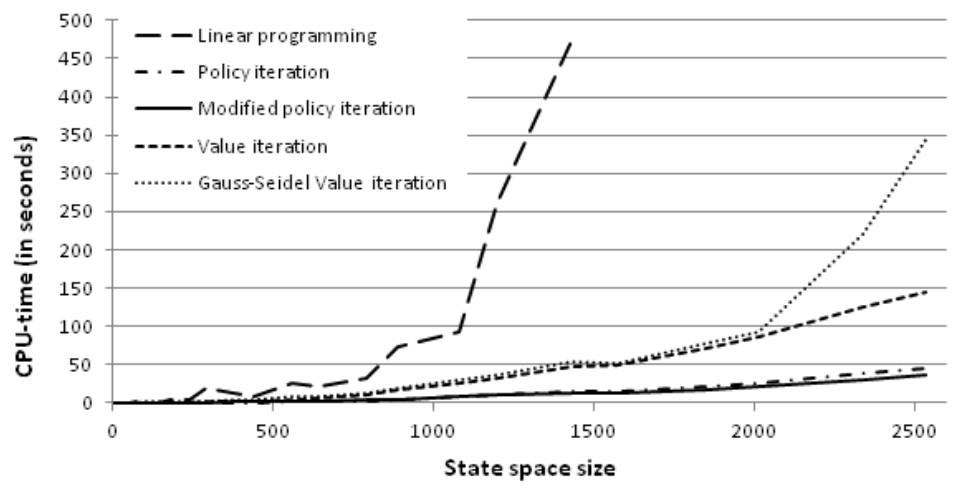

Fig. 7. A comparison between the different solution methods in terms of speed.

\section{Conclusion}

In this paper, we determine the optimal batch ordering policy for an inventory system with stochastic lead times, demand and price fluctuations by means of a versatile Markovian decision model. In the studied systems, there is at most one 
ongoing order and demand is of one unit. The optimal policy is determined under several price fluctuation scenarios such that a total cost function is minimised. The proposed cost function includes a holding cost, a fixed ordering cost, a variable purchase cost and a cost due to lost demand. The latter represents the risk that the demand is not satisfied due to an unexpected long lead time. As it becomes infeasible to solve a Markov decision process with a large state space by hand, several algorithms are used to find the optimal ordering policy.

As our numerical examples show, a relative small change in the parameters, e.g. the discount factor, the frequency and the variability of the price or the ordering cost, often has a major impact on the outcome of the model. This observation underlines the importance of a model customised for each company. Furthermore, a comparison between the different solution methods with respect to the state space is made. The research shows that the policy iteration algorithm, or a modified version of this algorithm, and secondly the value iteration algorithm present the best results for a system with such a large state space. However, the formulation of the problem as a linear programming problem is not appropriate. Finally, it can be stated that the required CPU time does not constitute an obstacle for solving our specific problem. The condition, however is to use a computer with enough RAM. This may also be solved by reducing the number of possible price levels or by limiting the number of possible batches.

\section{References}

1. Barbosa, L., Friedman, M.: Deterministic inventory lot size models Ũ a general root law. Management Science 24, 819-826 (1978)

2. Bellman, R.: A Markovian decision process. Journal of Mathematics and Mechanics 6(4), 679-684 (1957)

3. Chien, C.H., Wu, C.H., Chiang, Y.S.: Coordinated capacity migration and expansion planning for semiconductor manufacturing under demand uncertainties. INTERNATIONAL JOURNAL OF PRODUCTION ECONOMICS 135(2), 860-869 (FEB 2012)

4. Donaldson, W.: Inventory replenishment policy for a linear trend in demand: an analytical solution. Operational Research Quarterly 28, 663-670 (1977)

5. Harris, F.W.: How many parts to make at once. Factory, the magazine of management 10(2), 135-136 (1913)

6. Henery, R.: Inventory replenishment policy for increasing demand. Journal of the Operational Research Society, 30, 611-617 (1979)

7. Hillier, F.S., Lieberman, G.: Introduction to Operations Research. Chapter 19, McGraw-Hill, Singapore (2010)

8. Hopp, W., Spearman, M.: Factory Physics, Inventory Control: From EOQ to RDP. McGraw-Hill (2008)

9. Howard, R.: Dynamic programming and Markov processes. The M.I.T. Press (1960)

10. Miller, G.: Aggregate inventory management. PROACTION (2006)

11. Puterman, M., Shin, M.: Modified policy iteration algorithms for discounted Markov decision problems. Management Science 24 (1978) 
12. Resh, M., Friedman, M., Barbos, L.: On a general solution of the deterministic lot size problem with time-proportional demand. Operations Research 24, 718-725 (1976)

13. Song, J., Zipkin, P.: Inventory control in a fluctuating demand environment. Operations Research 41(2), 351-370 (March-April 1993)

14. Sutton, R.: On the significance of Markov decision processes. Environment 1327, 273-282 (1997)

15. Sutton, R., Barto, A.: Reinforcement learning: An introduction. Neural Networks, IEEE Transactions 9(5), 1054 (1998)

16. Teng, J.T., Chern, M.S., Chan, Y.L.: Deterministic inventory lot-size models with shortages for fluctuating demand and unit purchase cost. International Transactions in Operational Research 12(1), 83-100 (2005)

17. Tijms, H.C.: Stochastic modelling and analysis: A computational approach, Markovian decision processes and their application. John Wiley \& Sons Inc., New York (1986)

18. Yan, K., Kulkarni, V.: Optimal inventory policies under stochastic production and demand rates. Stochastic Models 24(2), 173-190 (2008) 